\title{
Instability of a Vacuum Arc Centrifuge
}

\author{
M. J. Hole, R. S. Dallaqua†, S. W. Simpson* and E. Del Bosco†. \\ EURATOM/UKAEA Fusion Association, Culham Science Centre, Abingdon, \\ Oxfordshire, OX14 3DB UK \\ *School of Electrical and Information Engineering, University of Sydney, N.S.W. 2006 Australia \\ $†$ Laboratório Associado de Plasma (LAP), Instituto Nacional de Pesquisas Espaciais (INPE), \\ CP 515, 12201-970, São José dos Campos, SP, Brazil
}

\begin{abstract}
Ever since conception of the Vacuum Arc Centrifuge (VAC) in 1980, periodic fluctuations in the ion saturation current and floating potential have been observed in Langmuir probe measurements in the rotation region of a VAC. Our theoretical and experimental research suggests that these fluctuations are in fact a pressure-gradient driven drift mode. In this work, we summarise the properties of a theoretical model describing the range of instabilities in the VAC plasma column, present theoretical predictions and compare with detailed experiments conducted on the PCEN centrifuge at the Brazilian National Space Research Institute (INPE). We conclude that the observed instability is a 'universal' instability, driven by the density-gradient, in a plasma with finite conductivity.
\end{abstract}

\section{INTRODUCTION}

In 1981, Krishnan, Geva and Hirshfield ${ }^{1}$ presented the first results for a new type of centrifuge, the vacuum arc centrifuge (VAC). The VAC is an axially configured plasma centrifuge, with a uniform plasma column present in a cylindrical vessel. Figure 1 shows a schematic of the PCEN centrifuge at the Brazilian National Space Research Institute (INPE) used for the experimental measurements reported here.

The plasma is created from metal vapour ablated from the cathode of the discharge by the action of vacuum arcs (see Figure 1) with a metal grid forming the anode of the discharge. The discharge is initiated by a high voltage pulse at the cathode. The interaction of the current with the axial magnetic field sets the plasma in rotation in the region to the left of the anode grid in the figure. ${ }^{2}$ The rotating metal vapor plasma streams supersonically through the anode mesh, passing through the rotation region and eventually impinging on the far endplate. Table 1 shows typical parameters of the VAC for the experiments with a magnesium cathode described here.

\begin{tabular}{lc} 
TABLE 1. Typical VAC Plasma Parameters & \\
\hline Parameter & value \\
\hline cathode & $\mathrm{Mg}$ \\
background gas pressure & $1.0 \times 10^{-4} \mathrm{~Pa}$ \\
mean ionization & 1.5 \\
axial magnetic field $\mathrm{B}_{\mathrm{z}}$ & $0.1 \mathrm{~T}$ \\
plasma temperature $\mathrm{T} \approx \mathrm{T}_{\mathrm{i}} \approx \mathrm{T}_{\mathrm{e}}$ & $5.5 \mathrm{eV}$ \\
discharge current & $1 \mathrm{kA}$ \\
discharge voltage & $5 \mathrm{kV}$ \\
plasma lifetime & $10 \mathrm{~ms}$ \\
on axis ion density in rotation region & $5.0 \times 10^{19} \mathrm{~m}^{-3}$ \\
characteristic column radius & $15 \mathrm{~mm}^{-1}$ \\
plasma rotation frequency & $150 \mathrm{krad} \mathrm{s}^{-1}$ \\
ion axial streaming velocity & $10^{4} \mathrm{~ms}^{-1}$ \\
\hline
\end{tabular}




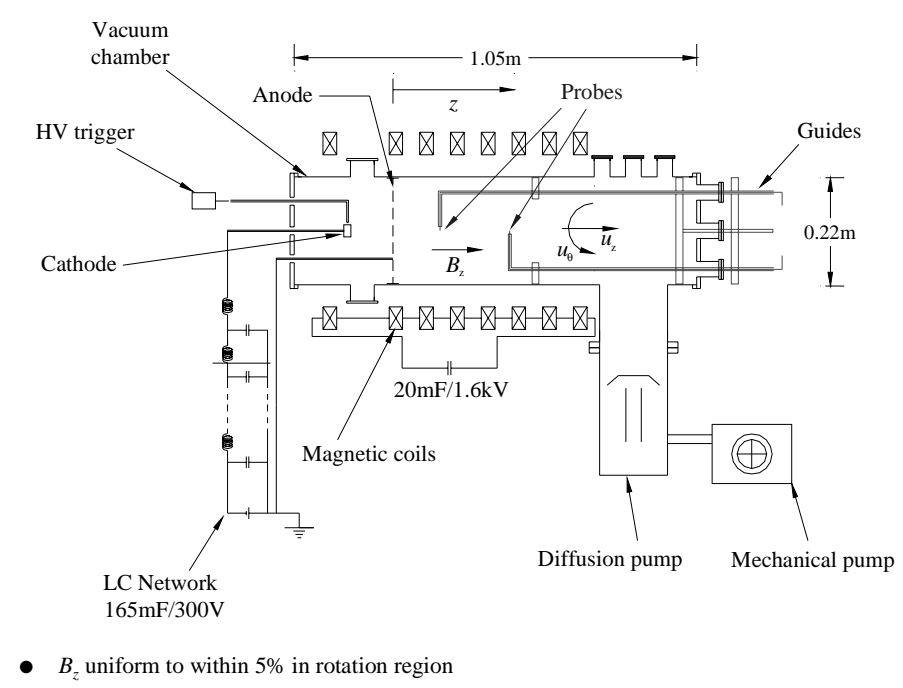

FIGURE 1. The PCEN vacuum arc centrifuge at the Brazilian National Space Research Institute (INPE).

Krishnan, Geva and Hirshfield ${ }^{1}$ and other researchers have made Langmuir probe measurements in the rotation region of vacuum arc centrifuges. A key feature of these measurements is the observation of periodic fluctuations in the ion saturation current and floating potential. Figure 2 shows typical oscillations.

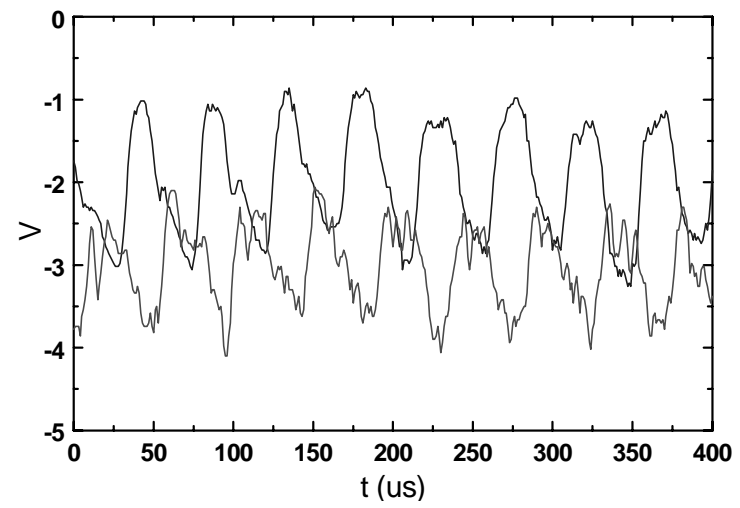

FIGURE 2. Time trace of floating potential: probes $180^{\circ}$ apart in azimuth, in rotation region of VAC.

This work presents a summary of experiments and a theoretical model aimed at explaining the observed oscillations. In the following section, the experiments are briefly described, and this is followed by an outline of the theory and comparison with experimental results.

\section{EXPERIMENT}

Most of the experimental measurements were made using Langmuir probes: the electron temperature Te was measured in the usual way by sweeping the probe bias voltage, and the ion density $\mathrm{n}_{\mathrm{i}}$ was deduced by measuring currents with the probe biased to the ion saturation voltage. To determine phase differences in the oscillation as a function of position in the plasma, a downstream probe at the edge of the plasma column was utilized to establish reference phase information. ${ }^{3}$ Figure 3 shows plasma properties as a function of radius across the plasma column.

\section{THEORY AND RESULTS}

The model is two-fluid in a cylindrical geometry ( $r, \theta, \mathrm{z}$ ), assuming a steady-state plasma which is azimuthally symmetric and has no axial structure in rigid rotation (see figure 3 ). The ion and electron temperatures are assumed uniform and the steady state ion density distribution has a Gaussian profile. Finite Larmor Radius (FLR) effects are neglected. Small perturbations are considered with unknown radial structure but standing waves in the $\theta$ direction and travelling in the $z$ direction. Long axial wavelength solutions with zero or small electron-ion collision frequency 
are investigated and the radial solutions are Generalised Laguerre polynomials in limiting cases, otherwise a finite difference numerical treatment is used.

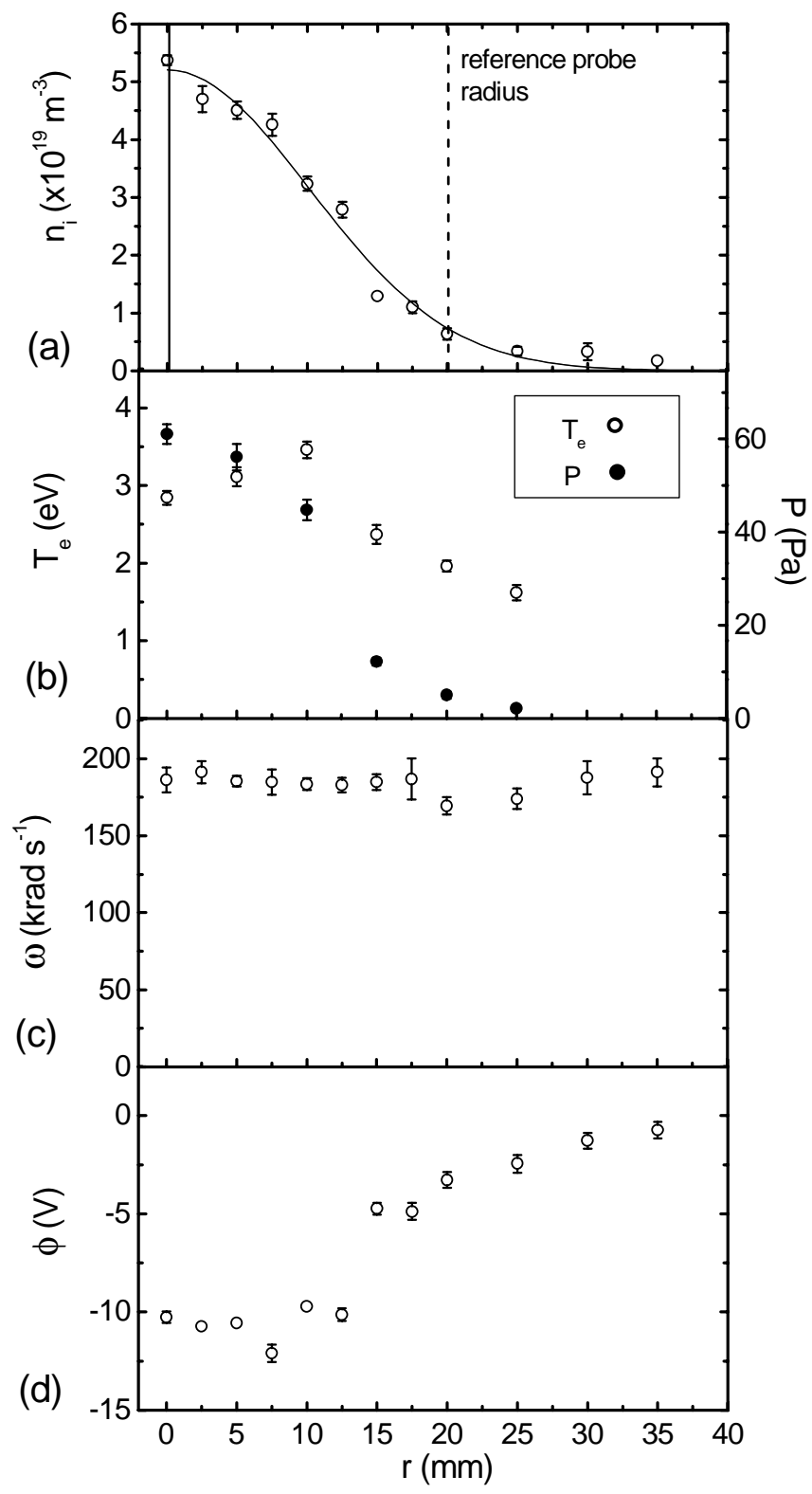

FIGURE 3. Radial profiles for $B_{z}=0.05 T$ and $z=150 \mathrm{~mm}$ (from grid) of (a) steady-state ion density, (b) electron temperature (left axis), and total pressure (right axis), (c) instability frequency and (d) steady-state floating potential.

Figures 4 and 5 compare experimental data with predictions for the density-gradient driven drift wave at maximum growth. The azimuthal mode number $\mathrm{m}$ is 1 . The wave is close to stationary in the frame of the ion fluid, and propagates downstream in the laboratory frame. The predicted slip of the wave (rotation speed of instability compared to plasma) is $20 \%$, which is comparable to the experimentally measured slip of $17 \%$. Figure 4 shows that the predicted amplitude profiles of the ion density oscillation and the floating potential oscillation are similar to measured profiles, while figure 5 indicates that the predicted radial variation of the phase difference between the ion 


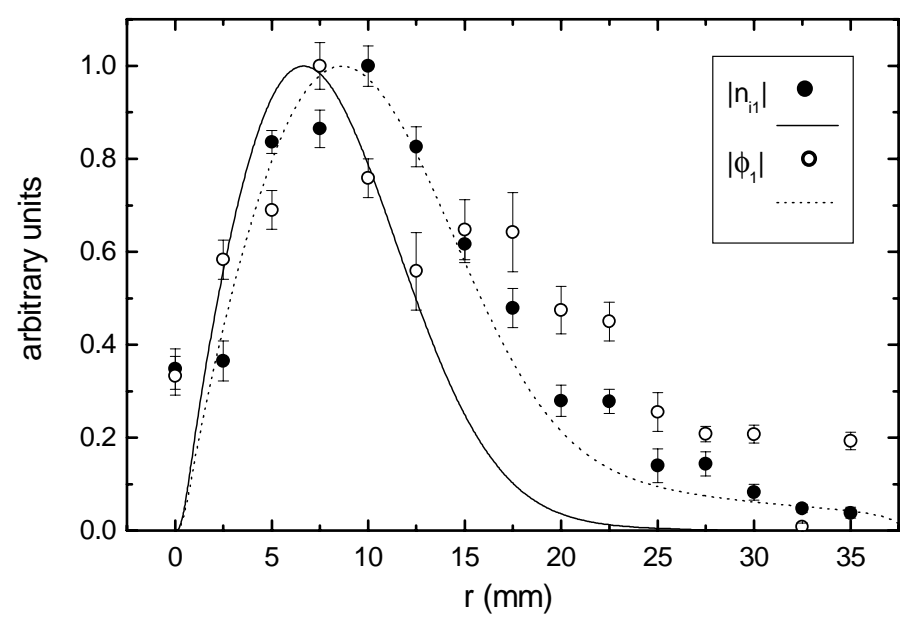

FIGURE 4. Circles are measurements of $\left|\mathrm{n}_{\mathrm{i} 1}\right|$ (solid) and $\left|\phi_{1}\right|$ (open). Lines are theory predictions of $\left|\mathrm{n}_{\mathrm{i}}\right|$ (solid) and $\left|\phi_{1}\right|$ (dotted). Measured and predicted amplitudes have been normalized to peak values.

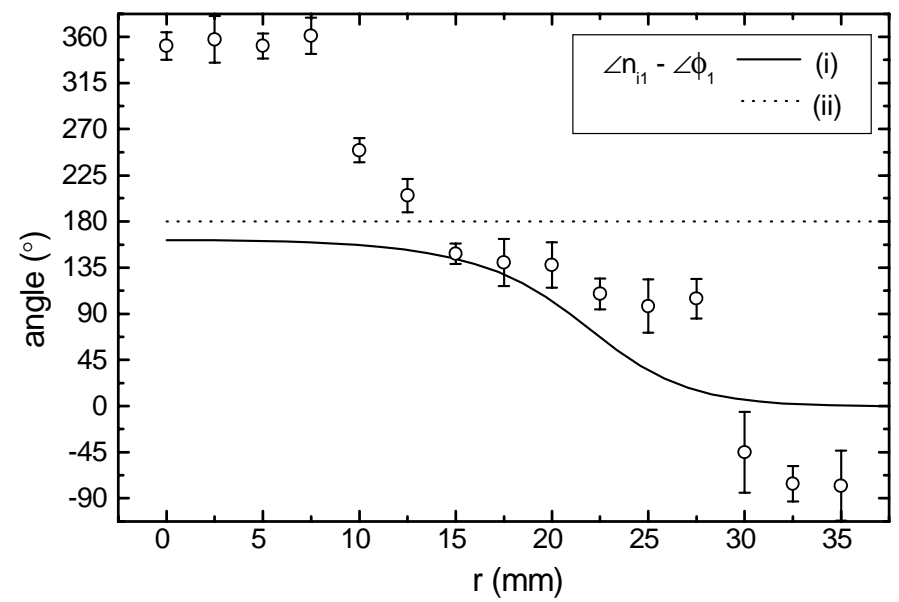

FIGURE 5. Circles are measurements of the phase of $n_{i 1}$ with respect to the phase of $\phi_{1}$. Lines are theory predictions of the phase difference for (i) the density gradient driven drift wave (solid) and (ii) the $(m, n)=(1,1)$ centrifugal instability.

density fluctuations and the floating potential fluctuations are also in satisfactory agreement with experiment except for radii less than $10 \mathrm{~mm}$. Possible explanations for the discrepancy are either an incomplete model or perturbation of the plasma by the probe. Figure 5 also plots the predicted phase difference for the centrifugal instability, which is not considered a likely candidate to explain the experimental data.

\section{ACKNOWLEDGMENTS}

The authors gratefully acknowledge the support of the Department of Education, Training and Youth Affairs, the School of Electrical and Information Engineering, and James King of Irrawang for the provision of Grants-in-aid that enabled one of us (MJH) to work in Brazil. The authors also gratefully acknowledge the financial support of the Research Foundation of the State of São Paulo (FAPESP) towards the PCEN experiment.

\section{REFERENCES}

1. Krishnan, M., Geva, M., and Hirshfield, J.L., Phys. Rev. Lett. 46, 36 (1981).

2. Simpson, S.W., Dallaqua, R.S., and Del Bosco, E., J. Phys. D.: Appl. Phys. 29, 1040 (1996).

3. Hole, M.J., Dallaqua, R., Simpson, S.W. and Del Bosco, E., Phys. Rev. E. 65, 046409-1 (2002). 